\title{
Palivizumab nella profilassi del virus respiratorio sinciziale: analisi di impatto sul budget del SSN italiano
}

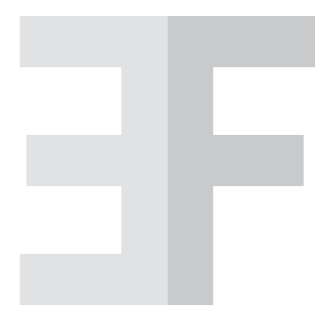

Daniela Roggeri ${ }^{(1)}$

\begin{abstract}
Palivizumab is a monoclonal antibody to RSV that has been shown to significantly reduce the frequency of hospitalisations for RSV infection, in at-risk populations. However, payers are concerned about the budgetary impact of adopting palivizumab. A budget impact model was developed to estimate the financial impact of palivizumab for the prevention of severe RSV infection in at-risk populations in the Netherlands. These analyses were adapted to Italy, after a brief review of the literature on the health care system and epidemiology of RSV infection in our country. The report below outlines the methodology and analysis of the costs associated with palivizumab prophylaxis of premature infants of 33 to 35 weeks gestational age which are the at-risk subgroup eligible for prophylaxis according to Italian guidelines.
\end{abstract}

Keywords: Budget impact; Palivizumab; RSV

Farmeconomia e percorsi terapeutici 2011; 12(Suppl 3): 17-23

\section{INTRODUZIONE}

L'efficacia clinica di palivizumab nella profilassi dei bambini ad alto rischio di infezioni severe da virus respiratorio sinciziale (RSV) è stata ampiamente supportata da diversi trial clinici [1-4]. Tuttavia, nell'ottica di un corretto utilizzo dei budget sanitari, per poter implementare coerentemente un programma di profilassi è necessario valutare anche i costi implicati nell'intervento. Nel caso della profilassi con palivizumab l'importanza di un'accurata analisi economica volta a valutare il rapporto di costoefficacia e l'impatto economico della profilassi diventa più che mai evidente. Il costo di profilassi è infatti piuttosto elevato e va attentamente valutato anche considerando gli outcome clinici favorevoli, soprattutto in termini di anni di vita guadagnati, di qualità della vita e di riduzione delle ospedalizzazioni e delle sequele da infezione da RSV.

\section{BACKGROUND}

I principali studi di valutazione economica su palivizumab condotti nella realtà italiana sono essenzialmente tre e comprendono due analisi di costo-efficacia $[5,6]$ e un'analisi di costo-utilità [7].

Lo studio di Ravasio e colleghi del 2006 ha valutato la costo-efficacia di palivizumab vs non profilassi in diverse categorie di bambini pretermine $(<33$ settimane di età gestazionale (wGa); $33<\mathrm{wGa}<35$ ) con o senza broncopneumodisplasia. L'analisi è stata condotta nella prospettiva del SSN italiano al fine di valutare gli anni di vita guadagnati (LYG), i QALY (anni di vita guadagnati ponderati per la qualità di vita) e i costi diretti sanitari comprendenti somministrazione (5 iniezioni di palivizumab da $15 \mathrm{mg} / \mathrm{kg}$ a distanza di 1 mese l'una dall'altra), ospedalizzazione e sequele a lungo termine [5]. In Figura 1 viene schematizzata la struttura del modello per quanto riguarda il ramo con profilassi nei bambini $<33 \mathrm{wGa}$; l'insorgenza o meno di sequele respiratorie (asma) viene valutata nei 13 anni successivi all'arruolamento. I dati di input del modello sono stati tratti da trial clinici internazionali e da costi e tariffe ospedaliere vigenti in Italia.

I risultati della simulazione hanno evidenziato in tutti i sottogruppi di rischio un guadagno medio di 0,088 LYG e 0,166 QALY nel ramo dei pazienti profilassati, rispetto al ramo che non ha ricevuto palivizumab. Il costo medio per paziente è risultato, sia nell' analisi generale sia nei tre sottogruppi, più elevato nel ramo profilassi a causa del costo elevato di palivizumab, che viene però parzialmente compensato dai minori costi per casi evitati (Tabella I). L'analisi ha inoltre evidenziato un costo incrementale della profilassi, rispetto alla non profilassi, pari a 19.813,08 $€ / L Y G$ e 10.524,21 €/QALY. Infine, l'analisi di (l) ProCure Solutions S.A.S, Nembro (BG)

Corresponding author

Daniela Roggeri

daniela_roggeri@procuresolutions.it 


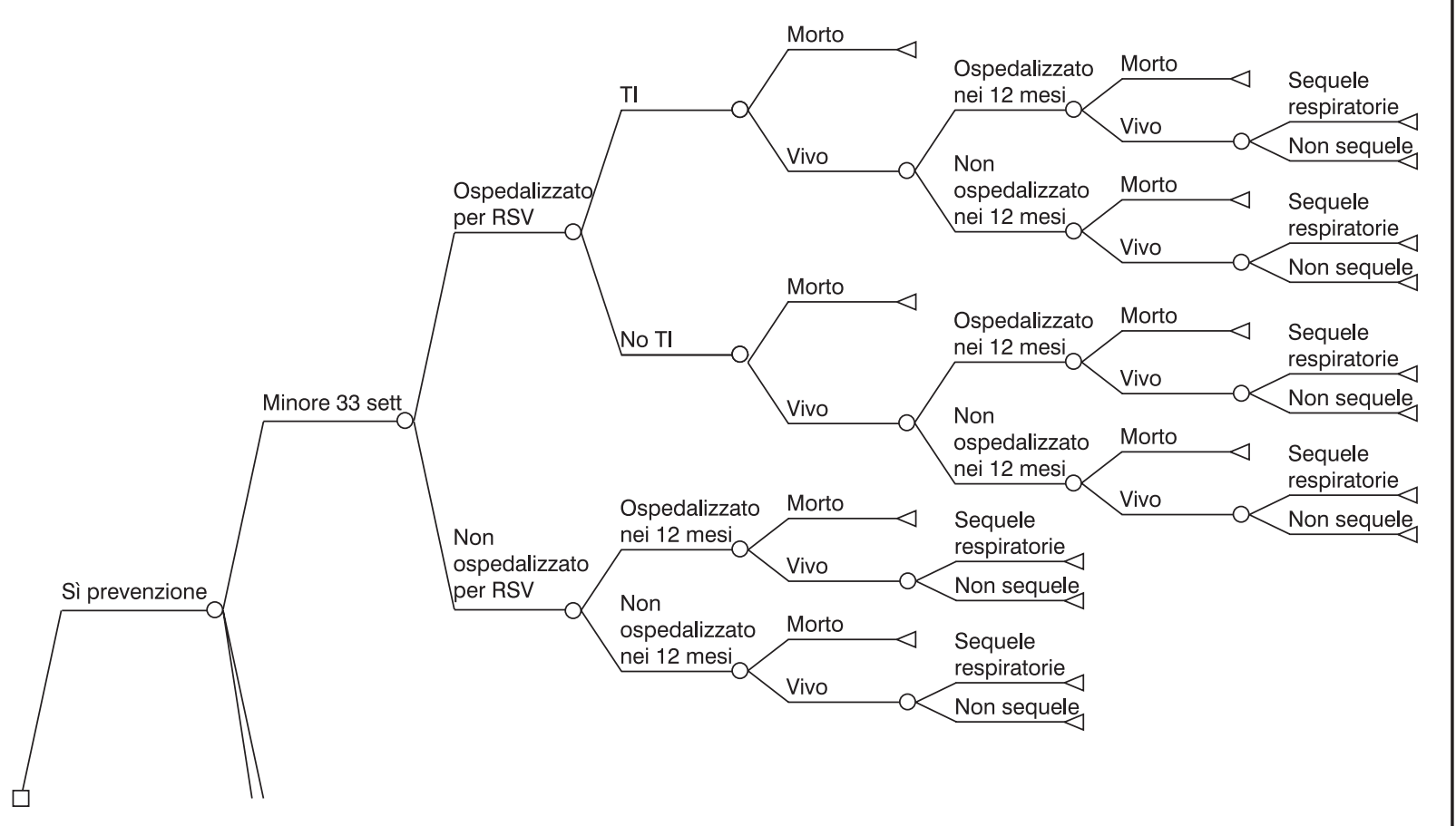

Figura 1

Struttura del modello per quanto riguarda il ramo con profilassi nei bambini < 33 wGa (gli altri due rami si sviluppano analogamente). Modificato da Ravasio, 2006 [5]

$\mathrm{TI}=$ terapia intensiva

soglia condotta in tutti e tre i sottogruppi ha confermato il favorevole rapporto di costo-efficacia della profilassi con palivizumab definendo accettabile l'investimento da sostenere per migliorare l'aspettativa e la qualità della vita.

La struttura del modello utilizzato nell'analisi di Ravasio [5] è stata successivamente utilizzata per una seconda valutazione economica condotta da Chirico e colleghi nel 2009 [6] e aggiornata sulla base dei risultati dello studio di Simoes e colleghi del 2007 [8]. Le differenze principali rispetto al modello originario sono:

- l'orizzonte temporale di 3 anni (due dopo l'arruolamento) invece di 14, al fine di allinearsi ai nuovi dati di letteratura utilizzati;

- lo schema di somministrazione di palivizumab considerato è di 3 fiale da $50 \mathrm{mg}$ e 2 fiale da $100 \mathrm{mg}$ (invece delle 5 fiale da $100 \mathrm{mg}$ considerate nell'analisi di Ravasio e colleghi [5]);

\begin{tabular}{lccc}
\hline & $\begin{array}{c}\text { Anni di vita } \\
\text { guadagnati (LYG) }\end{array}$ & QALY & $\begin{array}{c}\text { Costo medio } \\
(\mathbf{\epsilon} / \mathbf{p z})\end{array}$ \\
\hline Totale & $+0,088$ & $+0,166$ & $+1.744,22$ \\
BPD & $+0,122$ & $+0,231$ & $+677,36$ \\
$<33 \mathrm{wGa}$ & $+0,080$ & $+0,150$ & $+1.873,80$ \\
$33<\mathrm{wGa}<35$ & $+0,080$ & $+0,151$ & $+2.834,99$ \\
\hline
\end{tabular}

\section{Tabella I}

Differenza nei risultati tra il ramo in profilassi rispetto a quello non profilassi, in totale e nei tre sottogruppi di rischio (modificato da [5])

$\mathrm{BPD}=$ broncodisplasia polmonare; $w \mathrm{Ga}=$ settimana di età gestazionale
- il consumo di risorse sanitarie è stato valorizzato sulla base di costi e tariffe aggiornate al 2007.

I risultati del modello sono simili a quelli ottenuti in quello originario e confermano un aumento dei LYG e dei QALY nel ramo profilassi, rispetto a quello non profilassi $(+0,088 \mathrm{e}+0,159$, rispettivamente) e un aumento dei costi sanitari $(+28,3 \%)$ dovuti alla somministrazione di palivizumab. Come per il precedente modello, il guadagno in termini di anni di vita e di QALY legato all'utilizzo di palivizumab rende accettabili i costi incrementali sostenuti $(15.568,65$ $€ / L Y G$ e 8.676,74 €/QALY) [6].

Infine, l'analisi economica condotta da Chiroli e colleghi nel 2005 ha valutato la costo-efficacia della profilassi con palivizumab, in termini di anni di vita guadagnati, nel solo sottogruppo di bambini pretermine con cardiopatia congenita [7]. Il modello analitico decisionale è stato costruito sulla base di dati di efficacia derivanti da trial clinici, mentre le risorse utilizzate per la profilassi e il trattamento delle malattie respiratorie sono state valorizzate utilizzando tariffe nazionali e costi ospedalieri vigenti. I risultati hanno evidenziato un maggior costo/paziente, ma anche una maggiore aspettativa di vita, nel ramo profilassi ( $€ 3.990$ e 51,3 LYG) rispetto al ramo no profilassi ( $€ 596$ e 50,8 LYG). Il costo incrementale per ogni anno di vita guadagnato con la somministrazione di palivizumab è di $€ 7.186$, che viene ritenuto accettabile secondo la soglia di disponibilità a pagare italiana (pari a $60.000 € /$ LYG [9]) e pertanto determina la costo-efficacia 


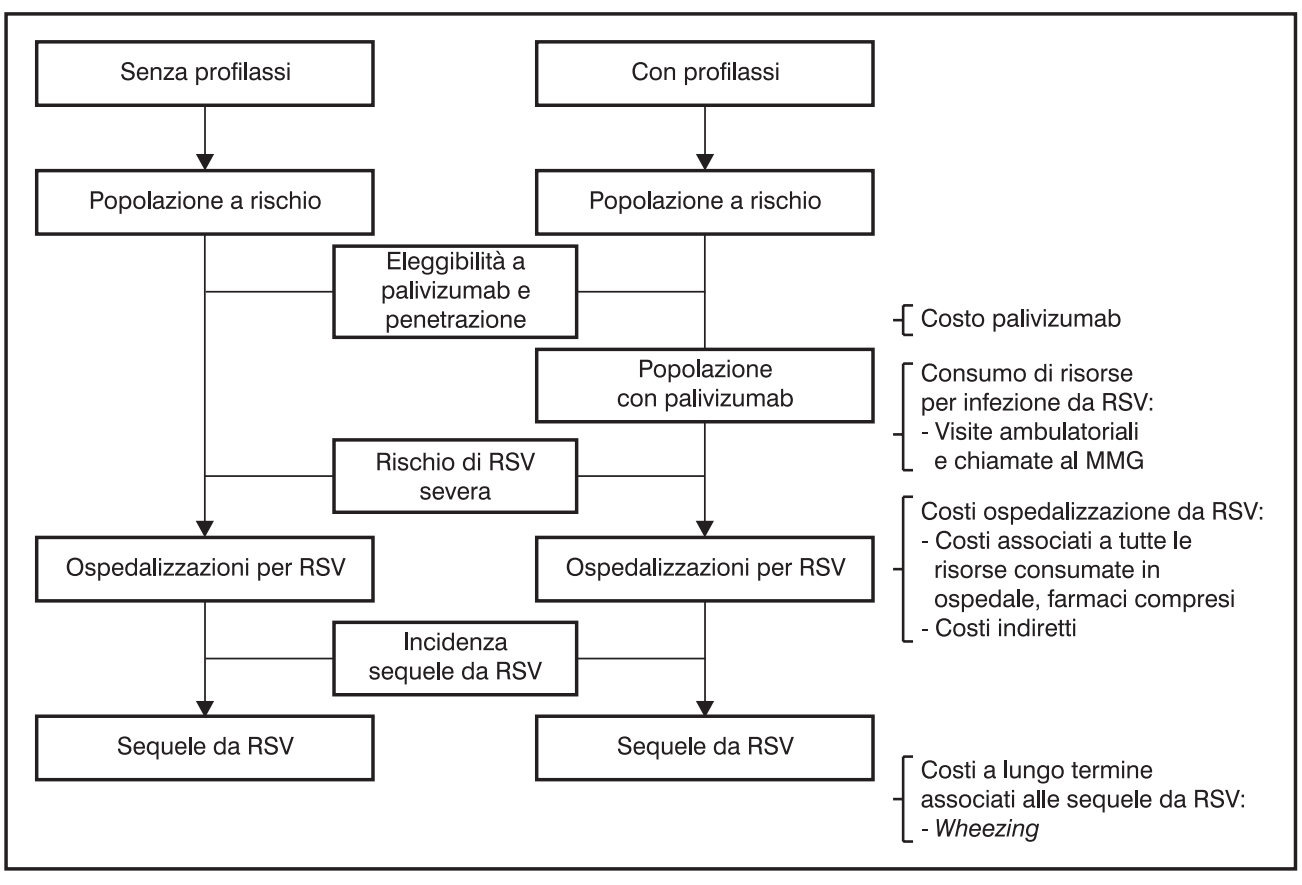

della profilassi con palivizumab nei bambini pretermine con cardiopatia congenita [7].

Gli studi finora presentati hanno valutato la costo-efficacia, in termini di LYG e QALY, della profilassi con palivizumab ma non vi sono considerazioni in merito al possibile impatto sul budget del SSN stimato attraverso un'analisi specifica che consideri tutti i costi implicati sia nella profilassi sia nel trattamento (in ospedale e fuori) delle malattie respiratorie e delle eventuali sequele a lungo termine dell'infezione da RSV. Obiettivo del presente lavoro è pertanto presentare l'adattamento alla realtà italiana di un modello di budget impact condotto sulla popolazione olandese, al fine di valutare l'impatto sul budget del SSN italiano dell'utilizzo di palivizumab nel sottogruppo di bambini pretermine con età gestazionale compresa tra le 33 e le 35 settimane, che è quello per cui le linee guida raccomandano la terapia solo in presenza di almeno due fattori di rischio [10].

\section{METODI}

Il modello è stato disegnato per essere incidence-based e per valutare l'impatto economico della profilassi con palivizumab su un orizzonte temporale di 3 anni. Il 2010 è stato scelto come anno di riferimento ed è stato stimato il budget impact nella prospettiva del Servizio Sanitario Nazionale italiano per gli anni 2011, 2012 e 2013. La struttura del modello è presentata in Figura 2 e considera due scenari principali: con e senza profilassi. Nel braccio senza profilassi sono state valutate le conseguenze cliniche e il conseguente impatto economico sulla base delle evidenze relative alla popolazione che non viene sottoposta a profilassi. Per quanto riguarda il braccio con profilassi è stato assunto che solamente un sottogruppo della popolazione a rischio ricevesse profilassi, così come indicato nelle più recenti linee guida della Società Italiana di Neonatologia [10].

\section{Popolazione target}

La popolazione target sulla quale impostare il modello è stata selezionata a partire dalla stima dei bambini nati vivi in Italia [11] alla quale è stato applicato il tasso di incidenza di nascita pretermine ( $<36$ settimane di età gestazionale), di diagnosi di broncodisplasia polmonare (BPD) e cardiopatia congenita emodinamicamente significativa (HS-CHD) nei primi due anni di vita [12] (Tabella II).

\begin{tabular}{lcccc}
\hline & $\begin{array}{c}\text { Anno base } \\
\mathbf{2 0 1 0}\end{array}$ & $\begin{array}{c}\text { Anno 1 } \\
\mathbf{2 0 1 1}\end{array}$ & $\begin{array}{c}\text { Anno 2 } \\
\mathbf{2 0 1 2}\end{array}$ & $\begin{array}{c}\text { Anno 3 } \\
\mathbf{2 0 1 3}\end{array}$ \\
\hline $\begin{array}{l}\text { Stima bambini nati vivi in Italia (n.) } \\
\text { Bambini prematuri senza BPD (n.) }\end{array}$ & 591.068 & 598.407 & 605.837 & 613.359 \\
$<28 \mathrm{wGa}$ & & & & \\
$29<\mathrm{wGa}<32$ & 5.200 & 5.252 & 5.305 & 5.358 \\
$33<\mathrm{wGa}<35$ & 8.500 & 8.585 & 8.671 & 8.758 \\
Bambini con BPD $($ n.) & 25.500 & 25.755 & 26.013 & 26.273 \\
Bambini con HS-CHD (n.) & 2.000 & 2.020 & 2.040 & 2.061 \\
\hline
\end{tabular}

Figura 2

Struttura del modello di budget impact

\section{Tabella II}

Popolazione a rischio di RSV severa in Italia negli anni considerati nell'analisi

$\mathrm{BPD}=$ broncodisplasia polmonare;

$\mathrm{HS}-\mathrm{CHD}=$ cardiopatia congenita emodinamicamente significativa $w G a=$ settimana di età gestazionale 


\begin{tabular}{lccc} 
& $\mathbf{2 0 1 1}$ & $\mathbf{2 0 1 2}$ & $\mathbf{2 0 1 3}$ \\
\hline Bambini prematuri $33<$ & $w \mathrm{Ga}<$ & & \\
$\%$ & 20 & 20 & eleggibili alla profilassi \\
N. & 5.151 & 5.203 & 20 \\
\hline
\end{tabular}

Tabella III

Stima dei bambini eleggibili alla profilassi con palivizumab nella categoria di rischio $33<w G a<35$

$w G a=$ settimana di età gestazionale

\begin{tabular}{lccc}
\hline & $\mathbf{2 0 1 1}$ & $\mathbf{2 0 1 2}$ & $\mathbf{2 0 1 3}$ \\
\hline Bambini prematuri $33<$ & wGa $<$ 35 sottoposti a profilassi \\
$\%$ & 50 & 52 & \\
N. & 2.576 & 2.705 & 55 \\
\hline
\end{tabular}

Tabella IV

Popolazione eleggibile che, secondo le stime di percentuale di penetrazione, riceve profilassi

$w G a=$ settimana di età gestazionale

\begin{tabular}{lcccc}
\hline & $\begin{array}{c}\text { Anno base } \\
\mathbf{2 0 1 0}\end{array}$ & $\begin{array}{c}\text { Anno 1 } \\
\text { 2011 }\end{array}$ & $\begin{array}{c}\text { Anno 2 } \\
\text { 2012 }\end{array}$ & $\begin{array}{c}\text { Anno 3 } \\
\mathbf{2 0 1 3}\end{array}$ \\
\hline Totale popolazione a rischio (n.) & 25.500 & 25.755 & 26.013 & 26.273 \\
$\begin{array}{l}\text { Totale popolazione eleggibile } \\
\text { alla profilassi (n.) }\end{array}$ & - & 5.151 & 5.203 & 5.255 \\
$\begin{array}{l}\text { Totale popolazione eleggibile } \\
\text { che riceve profilassi (n.) }\end{array}$ & - & 2.576 & 2.705 & 2.890 \\
\hline
\end{tabular}

\section{Tabella V}

Riassunto popolazione a rischio $33<w G a<35$, eleggibile e profilassata considerata nel modello

\begin{tabular}{lcc}
\hline & Senza profilassi & Con profilassi \\
\hline Tassi di ospedalizzazione & 0,081 & 0,018 \\
Tasso di re-ospedalizzazione & 0,38 & 0,38 \\
Tasso di incidenza sequele (wheezing) & 0,191 & 0,068 \\
\hline
\end{tabular}

\section{Tabella V}

Tassi di ospedalizzazione, di re-ospedalizzazione e di incidenza di sequele considerati nel modello

\section{Popolazione profilassata}

Ovviamente non tutti i bambini a rischio candidabili alla profilassi con palivizumab vengono poi effettivamente profilassati. La percentuale di penetrazione della profilassi nella categoria di rischio $33<\mathrm{wGa}<35$ adottata nel modello è stata indicata dall'azienda che commercializza palivizumab in Italia [12]. Il numero di bambini che si è ipotizzato ricevano palivizumab negli anni in analisi (Tabella IV) è stato calcolato moltiplicando il numero di bambini eleggibili alla profilassi (Tabella III) per la percentuale di penetrazione.

In Tabella $\mathrm{V}$ viene riassunto, per ognuno degli anni in analisi nel modello, il numero totale di soggetti a rischio, la popolazione eleggibile alla profilassi e i bambini che effettivamente vengono profilassati.

\section{Ospedalizzazione e tasso di sequele}

Il tasso di ospedalizzazione nella categoria di rischio $33<\mathrm{wGa}<35$, con e senza profilassi, è stato tratto dal trial clinico IMpact-RSV trial [1]. Per quanto riguarda le sequele a lungo termine dell'infezione severa da RSV è stato preso in considerazione solo il wheezing ed è stato stimato un tasso di incidenza pari a 0,191 nei bambini che non ricevono profilassi e di 0,068 tra quelli profilassati [8]. Infine il rischio di re-ospedalizzazione negli anni successivi nei bambini già ospedalizzati per infezione da RSV è stato considerato pari a 0,38 [13] (Tabella VI).

\section{Consumo di risorse e costi}

\section{Costi di profilassi}

I costi associati alla profilassi comprendono unicamente il costo di palivizumab $(€ 814$ per fiala da $100 \mathrm{mg}$, come da Listino prezzi Abbott [14]), mentre il costo di somministrazione non viene conteggiato in quanto avviene in ospedale ed è pertanto incluso nello stipendio dell'operatore sanitario che la effettua. Il costo totale della profilassi dipende sostanzialmente dal numero di dosi somministrate, dal peso medio del bambino e dal numero di bambini che ricevono la profilassi. I dati relativi al peso medio alla nascita sono dati forniti dalla ditta produttrice del farmaco [12]. Il peso medio dei bambini all'inizio e al termine della profilassi è stato calcolato applicando un incremento medio di $105 \mathrm{~g} /$ settimana al peso medio alla nascita [15]. In Tabella VII vengono riportati i costi unitari e totali della profilassi con palivizumab (Euro 2010).

\section{Costi di ospedalizzazione}

Il costo medio per ricoveri da RSV è stato stimato come costo medio ponderato per la numerosità dei ricoveri dei 3 DRG associati alle nascite premature [16] (Tabella VIII). Il costo di ogni ricovero è stato stimato utilizzando la tariffa unica convenzionale (TUC) 2010 [17]. 


\section{Costo post-dimissione}

La stima delle risorse consumate dai pazienti dopo le dimissioni dall'ospedale è principalmente basata su dati di letteratura, mentre i relativi costi unitari sono stati estrapolati da alcuni studi economici condotti in merito [18$20]$. Le categorie di costo considerate sono le chiamate a domicilio e le visita dal pediatra e gli ingressi al pronto soccorso, per un costo totale post-dimissione pari a $€ 147$ paziente/anno.

\section{Costi sequele a lungo termine da RSV}

Nel modello l'unica sequela a lungo termine considerata è il wheezing e, in assenza di dati di costo specifici alla realtà italiana, è stato utilizzato un dato derivante da uno studio tedesco del 2009 [21] e attualizzato al 2010 (secondo un tasso di inflazione del $6 \%$ [22]). Ne risulta un costo per il trattamento delle sequele a lungo termine da RSV pari a $1.037 € /$ anno.

\section{Assunzioni del modello}

Nella costruzione del modello sono state effettuate alcune assunzioni di seguito riportate:

- tutti i soggetti in analisi sono coperti dal SSN;

- per i bambini ospedalizzati per infezione da RSV, è stato ipotizzato un aumento del $38 \%$ del rischio di re-infezione durante la stagione successiva [13];

- non è stato considerato alcun costo di somministrazione di palivizumab, in quanto tale costo è stato considerato incluso nello stipendio dell'infermiere;

- il costo di palivizumab è stato considerato pari a $€ 814$ per ogni fiala da $100 \mathrm{mg}$ [14];

- i costi sono riferiti al 2010 e non si è tenuto conto di eventuale inflazione per il calcolo dei costi degli anni successivi;

- sono stati considerati solo i costi a lungo termine associati alle sequele dell'RSV;

- la durata della stagione di RSV è stata considerata di cinque mesi per tutti gli anni in analisi;

- sono state considerate fino a 5 iniezioni di palivizumab per ciclo di trattamento;

- tutti i pazienti hanno mostrato completa aderenza alla profilassi.

\begin{tabular}{lc}
\hline Consumo medio di fiale $(\mathrm{n})$. & 4,00 \\
Costo medio per fiala $(€)$ & 814 \\
Peso medio del bambino $(\mathrm{kg})$ & \\
$\quad$ Inizio profilassi & 3,22 \\
$\quad$ Fine profilassi & 5,05 \\
Costo medio di profilassi (€) & 3.257 \\
\hline
\end{tabular}

\section{Tabella VII}

Costi unitari e totali (espressi in $€$, anno di riferimento 2010) della profilassi con palivizumab

\section{RISULTATI}

In Tabella IX viene riportato il budget impact per i tre anni considerati nell'analisi ( 2011 2012 e 2013) relativo al sottogruppo di bambini pretermine con età gestazionale compresa tra 33 e 35 settimane. In questa sottopopolazione per il 2010 (senza profilassi) è stato stimato un costo totale a carico del SSN per le infezioni severe da RSV che richiedono ospedalizzazione pari a $€ 15.290 .913$, di cui $€ 14.578 .299$ imputabili all'ospedalizzazione. La spesa del SSN nell'anno 1 risulterebbe pari a $€ 15.443 .822$ nello scenario senza profilassi $v s € 22.626 .158$ in quello con profilassi (162 ospedalizzazioni evitate). Analogamente per gli anni successivi è stato stimato un impatto sul budget pari $€$ 21.466 .912 nell'ipotesi senza profilassi vs $€$ 28.554.787 nell'ipotesi con profilassi nell'anno 2 e $€ 21.681 .581$ vs $€ 29.261 .497$ nell'anno 3 ; la profilassi consentirebbe di evitare al secondo e terzo anno rispettivamente 232 e 247 ricoveri.

In Figura 3 vengono riportati i costi di ospedalizzazione sostenuti nei due scenari di analisi.

\section{DISCUSSIONE E CONCLUSIONI}

L'analisi qui presentata, adattamento italiano di una valutazione economica condotta sulla popolazione olandese, ha valutato l'impatto sul budget del SSN dell'adozione della profilassi con palivizumab in bambini ad alto rischio di infezione severa da RSV. In particolare, è stata condotta un'analisi mirata nel sottogruppo a rischio rappresentato dai bambini pretermine con età gestazionale compresa tra 33 e 35 settimane, che è quello per cui le linee guida raccomandano la terapia. Nel corso del terzo anno dopo l'implementazione del programma

\begin{tabular}{lccc}
\hline \multicolumn{1}{c}{ DRG } & $\begin{array}{c}\text { Ricoveri (n.) } \\
\text { [16] }\end{array}$ & $\begin{array}{c}\text { Percentuale } \\
\text { dimessi }\end{array}$ & $\begin{array}{c}\text { Tariffe TUC } \\
\text { 2010 (€) [17] }\end{array}$ \\
\hline $\begin{array}{l}\text { 386 - Neonati gravemente immaturi o con sindrome } \\
\text { da distress respiratorio }\end{array}$ & 8.289 & $22 \%$ & 13.916 \\
387 - Prematurità con affezioni maggiori & 8.923 & $24 \%$ & 7.760 \\
388 - Prematurità senza affezioni maggiori & 20.062 & $54 \%$ & 3.913 \\
Costo medio ponderato per ospedalizzazione $(€)$ & & & 7.058 \\
\hline
\end{tabular}

\section{Tabella VIII}

Costo medio di ospedalizzazione per infezione da RSV

TUC = Tariffa Unica

Convenzionale 


\begin{tabular}{|c|c|c|c|c|c|}
\hline & & \multicolumn{2}{|c|}{ Senza profilassi } & \multicolumn{2}{|c|}{ Con profilassi } \\
\hline & & N. & Costo totale $(€)$ & N. & Costo totale $(€)$ \\
\hline & \multicolumn{5}{|l|}{ Anno base -2010} \\
\hline & Profilassi & - & - & - & - \\
\hline & Ospedalizzazioni & 2.066 & 14.578 .299 & - & - \\
\hline & Visite post-dimissioni & 2.066 & 303.629 & - & - \\
\hline & Sequele & 395 & 408.985 & - & - \\
\hline & Totale & 25.500 & 15.290 .913 & - & - \\
\hline & \multicolumn{5}{|l|}{ Anno 1-2011 } \\
\hline & Profilassi & - & - & 2.576 & 8.389 .434 \\
\hline & Ospedalizzazioni & 2.086 & 14.724 .082 & 1.924 & 13.578 .876 \\
\hline & Visite post-dimissioni & 2.086 & 306.665 & 1.924 & 282.813 \\
\hline & Sequele & 398 & 413.075 & 362 & 375.036 \\
\hline & Totale & 25.755 & 15.443 .822 & 25.755 & 22.626 .158 \\
\hline & \multicolumn{5}{|l|}{ Budget impact } \\
\hline & Inclusi i costi di profilassi & & & & 7.182 .336 \\
\hline & Esclusi i costi di profilassi & & & & -1.207 .098 \\
\hline & \multicolumn{5}{|l|}{ Anno 2 - 2012} \\
\hline & Profilassi & - & - & 2.705 & 8.812 .261 \\
\hline & Ospedalizzazioni & 2.900 & 20.466 .474 & 2.668 & 18.828 .371 \\
\hline & Visite post-dimissioni & 2.900 & 426.264 & 2.668 & 392.147 \\
\hline & Sequele & 554 & 574.174 & 504 & 522.009 \\
\hline & Totale & 26.013 & 21.466 .912 & 26.013 & 28.554 .787 \\
\hline & \multicolumn{5}{|l|}{ Budget impact } \\
\hline & Inclusi i costi di profilassi & & & & 7.087 .875 \\
\hline & Esclusi i costi di profilassi & & & & -1.724 .386 \\
\hline & \multicolumn{5}{|l|}{ Anno 3-2013 } \\
\hline & Profilassi & - & - & 2.890 & 9.413 .867 \\
\hline & Ospedalizzazioni & 2.929 & 20.671 .139 & 2.682 & 18.928 .980 \\
\hline & Visite post-dimissioni & 2.929 & 430.527 & 2.682 & 394.242 \\
\hline \multirow{5}{*}{$\begin{array}{l}\text { Tabella IX } \\
\text { Budget impact annuale } \\
\text { nello scenario base per } \\
\text { il sottogruppo di bambini } \\
\text { a rischio con età } \\
\text { gestazionale compresa } \\
\text { tra } 33 \text { e } 35 \text { settimane }\end{array}$} & Sequele & 559 & 579.916 & 506 & 524.408 \\
\hline & Totale & 26.273 & 21.681 .581 & 26.273 & 29.261 .497 \\
\hline & Budget impact & & & & \\
\hline & Inclusi i costi di profilassi & & & & 7.579 .915 \\
\hline & Esclusi i costi di profilassi & & & & -1.833 .952 \\
\hline
\end{tabular}

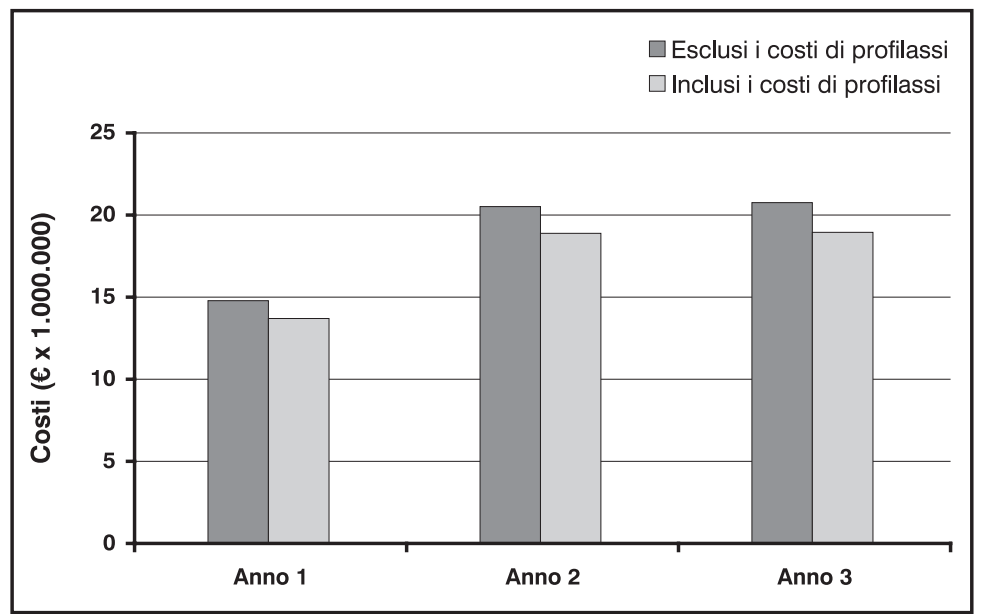

Figura 3

Costi di ospedalizzazione nei due scenari nel corso dei tre anni di analisi nel sottogruppo di bambini con età gestazionale compresa tra 33 e 35 settimane di profilassi, la somministrazione di palivizumab ai soggetti a rischio comporta un investimento aggiuntivo di risorse sanitarie pari a $€$ 7.579.915 a fronte di una riduzione delle ospedalizzazioni per infezione severa da RSV e dei costi a esse correlate ( -247 ospedalizzazioni e -1.742 .159 euro).

Alla luce dei risultati della presente analisi è possibile concludere che, sebbene l'investimento richiesto al SSN per attuare il programma di profilassi con palivizumab nei bambini a rischio di infezione severa da RSV possa sembrare elevato, uno sguardo più attento $\mathrm{e}$ critico alle ospedalizzazioni e ai costi da esse derivanti, che vengono sensibilmente ridotti nel corso dei tre anni di implementazione dell'intervento profilattico, induce a giustificare la spesa sostenuta. 


\section{BIBLIOGRAFIA}

1. The IMpact-RSV Study Group. Palivizumab, a humanized Respiratory Syncytial Virus monoclonal antibody, reduces hospitalization from respiratory syncytial virus infection in high-risk infants. Pediatrics 1998; 102: 531-7

2. Feltes TF, Cabalka AK, Meissner HC, Piazza FM, Carlin DA, Top FH, for the Cardiac Synagis Study Group. Palivizumab prophylaxis reduces hospitalization due to Respiratory Syncytial Virus in young children with hemodynamically significant congenital heart disease. J Pediatr 2003; 143: 532-40

3. Subramanian KN, Weisman LE, Rhodes T, Ariagno R, Sánchez PJ, Steichen J et al. Safety, tolerance and pharmacokinetics of a humanized monoclonal antibody to respiratory syncytial virus in premature infants and infants with bronchopulmonary dysplasia. MEDI-493 Study Group. Pediatr Infect Dis J 1998; 17: 110-5

4. Sáez-Llorens X, Castaño E, Null D, Steichen J, Sánchez PJ, Ramilo O et al. Safety and pharmacokinetics of an intramuscular humanized monoclonal antibody to respiratory syncytial virus in premature infants and infants with bronchopulmonary dysplasia. The MEDI-493 Study Group. Pediatr Infect Dis J 1998; 17: 787-91

5. Ravasio R, Lucioni C, Chirico G. Costo-efficacia di palivizumab versus non profilassi nella prevenzione delle infezioni da VRS nei bambini pretermine, a diversa età gestazionale. Pharmacoeconomics Ital Res Articles 2006; 8: 105-17

6. Chirico G, Ravasio R, Sbarigia U. Cost-utility analysis of palivizumab in Italy: results from a simulation model in the prophylaxis of respiratory syncytial virus infection (RSV) among high-risk preterm infants. Ital J Pediatr 2009; 35: 4

7. Chiroli S, Macagno F, Lucioni C. Cost-efficacy analysis of palivizumab in the prevention of respiratory syncytial virus infections in young children with hemodynamically significant congenital heart disease. Ital J Pediatr 2005; 31: $188-94$

8. Simoes EA, Groothuis JR, Carbonell-Estrany X, Rieger CH, Mitchell I, Fredrick LM et al. Palivizumab prophylaxis, respiratory syncytial virus, and subsequent recurrent wheezing. J Pediatr 2007; 151: 34-42

9. Messori A, Santarlasci B, Trippoli S, Vaiani M. Controvalore economico del farmaco e beneficio clinico: stato dell'arte della metodologia ed applicazione di un algoritmo farmacoeconomico. Pharmacoeconomics Ital Res Articles 2003; 5: 3-17

10. Consiglio Direttivo della Società Italiana di Neonatologia. Raccomandazioni della Società Italiana di neonatologia per la prevenzione delle malattie da virus respiratorio sinciziale (VRS). Aggiornamento 2004. Acta Neonatologica \& Pediatrica 2004; 1: 19-29

11. Projected live births. Statistiche italiane 2010. Disponibile on line all'indirizzo http://demo.istat.it/altridati/IscrittiNascita/index_e.html (ultimo accesso maggio 2011)

12. Abbott Italy affiliate. Abbott personal communication. 2010

13. McCormick MC, Shapiro S, Startfield BH. Rehospitalization in the first year of life for high-risk survivors. Pediatrics $1980 ; 66$; 991-9

14. Listino Ufficiale Abbott srl

15. Curve di crescita per prematuri. Pediatra on Web, 2010. Disponibile on line all'indirizzo http://www.dottorbedendo. it/crescita_curve_prematuri_1500.htm (ultimo accesso maggio 2011)

16. Ministero della Salute. Rapporto annuale sull'attività di ricovero ospedaliero. Dati SDO 2009. Disponibile on line all'indirizzo http://www.salute.gov.it/imgs/C_17_pubblicazioni_1491_allegato.pdf (ultimo accesso maggio 2011)

17. Tariffa Unica Convenzionale (TUC) per le prestazioni di assistenza ospedaliera per acuti. Anno 2010

18. Accordini S, Bugiani M, Arossa W, Gerzeli S, Marinoni A, Olivieri M et al. Poor control increases the economic cost of asthma. A multicentre population-based study. Int Arch Allergy Immunol 2006; 141: 189-98

19. Dal Negro R, Piskorz P, Vives R, Guilera M, Sazonov Kocevar V, Badia X. Healthcare utilisation and costs associated with adding montelukast to current therapy in patients with mild to moderate asthma and co-morbid allergic rhinitis: PRAACTICAL study. Pharmacoeconomics 2007; 25: 665-76

20. Paramore LC, Mahadevia PJ, Piedra PA. Outpatient RSV lower respiratory infections among high-risk infants and other pediatric populations. Pediatr Pulmonol 2010; 45: 578-84

21. Nuijten M, Lebmeier M, Wittenberg W. Cost effectiveness of palivizumab in children with congenital heart disease in Germany. J Med Econ 2009; 12: 301-8

22. The European Central Bank. Harmonised indicies of consumer prices, breakdown by purpose of consumption: 1.7 Health. ECB 2010 Disponibile on line all'indirizzo http:/www.ecb.europa.eu/stats/prices/hicp/html/index.en.html (ultimo accesso maggio 2011) 